\title{
Pensamiento Computacional: Revisión de experiencias y propuestas educativas implementadas en la última década en Iberoamérica
}

\author{
Computational Thinking: review of experiences and educational proposals implemented in \\ the last decade in latin america
}

Maira Isbeth Sarmiento Bolívar ${ }^{1}$

https://orcid.org/0000-0001-9066-2726

Universidad Pedagógica Nacional, Colombia

Recibido: 10-10-2019

Aceptado: 03-12-2019

\section{Cita ReCOMENDADA}

Sarmiento, M., (2019). Pensamiento computacional: Revisión de experiencias y propuestas educativas implementadas en la última década en Iberoamérica. Hamut'ay, 6(3), 26-36.

http://dx.doi.org/10.21503/hamu.v6i3.1842

\section{RESUMEN}

El siguiente artículo presenta la revisión realizada a 50 experiencias encontradas en Iberoamérica, cuyo objetivo es fomentar las competencias inherentes al pensamiento computacional a través de actividades de programación o temas relacionados, de las 50 propuestas se analizan 37 minuciosamente, porque abordan el grupo objeto de estudio, en este caso estudiantes de educación básica, media y primeros años de universidad; cuentan con información acerca de la implementación, las estrategias utilizadas y los resultados obtenidos, de este modo se pueden conocer aspectos en la realización de las mismas. Para encontrar la información referente a este tema se siguió un esquema de búsqueda y revisión de documentos publicados en internet y otras fuentes documentales relacionadas (revistas en línea, tesis, entre otros). Con base en la información se hizo un análisis comparativo que mostró características de las experiencias, siendo Argentina el país con mayor cantidad de iniciativas con un $32 \%$; el tipo de herramientas software y hardware utilizado en un $50 \%$ es de diseño propio, junto con herramientas libres; la zona de mayor aplicación fue institucional con un 62\%; el nivel educativo con mayor implementación fue el secundario; así como otros aspectos relacionados con la puesta en marcha de las propuestas. Se concluye que hay diversas estrategias que se pueden utilizar para promover el pensamiento computacional, la información a la que se hace referencia en este artículo sirve como orientación en el diseńo de nuevas propuestas adaptadas a los entornos educativos en los cuales se quieren implementar.

Palabras Clave: Enseñanza de la programación, educación en tecnología, pensamiento computacional, competencias tecnológicas, entornos de programación.

1 Magister y Especialista en Tecnología Informática Aplicada en Educación, ambos por la Universidad Nacional de La Plata (UNLP), Argentina. Especialista en Tecnologías de Información Aplicadas en Educación, y Licenciada en Diseńo Tecnológico, Universidad Pedagógica Nacional (UPN), Colombia. Se desempeña como docente y jefe del área de Tecnología e Informática en la Institución Educativa Departamental Pío XII, municipio de Pacho, Cundinamarca, Colombia. may1740@gmail.com 


\begin{abstract}
The following article presents the review carried out on 50 experiences found in Iberoamerica, whose objective is to promote the competences inherent to computational thinking through computer programming activities or related topics. Of the 50 proposals, we analyzed 37 thoroughly, because they address the target group, in this case students from basic and middle education and students from the first years of university. They have information about the execution, the strategies used and the results obtained, in this way we can know aspects about their implementation. To find information regarding this topic, we followed a search scheme and review of documents published on the Internet and other related documentary sources (online journals, thesis, among others). Based on the information, we made a comparative analysis that showed characteristics of the experiences, such as: the country with the largest number of initiatives, is Argentina with 32\%; the type of software and hardware tools used by $50 \%$ is of their own design, along with free tools; the area of the largest application was institutional with a $62 \%$; the educational level with the greatest implementation was secondary; as well as other aspects related to the implementation of the proposals. It is concluded that there are various strategies that can be used to promote computational thinking. The information referred in this article, serves as a guidance in the design of new proposals adapted to the educational environments in which they want to be implemented.
\end{abstract}

Keywords: Computer programming teaching, technology education, computational thinking, technological skills, computer programming environments.

\section{INTRODUCCIÓN}

Hoy en día, la integración y uso de las tecnologías como herramientas que favorecen los procesos de enseñanza y de aprendizaje en las diferentes asignaturas ha ganado terreno, por este motivo se implementan diversos proyectos impulsados desde políticas públicas alrededor de Iberoamérica, como es el caso de Computadores Para Educar en Colombia y Plan Ceibal en Uruguay, entre otros. Estas iniciativas reconocen la importancia de fomentar las competencias necesarias para desempeñarse en la sociedad del conocimiento, designadas por la UNESCO como competencias del siglo XXI, por este motivo buscan generar un impacto favorable brindando acceso de este tipo de tecnologías a la mayor cantidad de estudiantes posible, reduciendo así la brecha digital existente, cuestión que se convierte en un desafío a abordar en los procesos de integración que se vienen gestando (SITEAL, 2014).

Al reconocerse el valor de los proyectos y de las experiencias vinculadas a estos, sobre todo aquellas que fomentan el pensamiento computacional, se hace importante explorar y profundizar las características de estas propuestas, indagar los escenarios educativos en los que se han desarrollado, revisar el tipo de hardware y software utilizado, reconocer los materiales diseñados para su implementación y saber desde que enfoque se propone desarrollar las competencias pertenecientes al pensamiento computacional, con el fin de conocer las fortalezas de las estrategias utilizadas y contar con un referente para desarrollar nuevas propuestas. En esta revisión bibliográfica se realizó el análisis de experiencias desarrolladas en Iberoamérica con el objetivo de conocer las estrategias y métodos utilizados para fomentar el pensamiento computacional, por este motivo se debió adoptar un concepto acerca de este tipo de pensamiento.

\section{Pensamiento computacional}

Al realizar la revisión de documentos relacionados con el concepto y las características referentes al pensamiento computacional se encuentran diversas acepciones tanto en ámbitos pedagógi- 
cos como en ámbitos científicos, para encontrar información al respecto se puede revisar el trabajo de investigación: Análisis de experiencias y estrategias educativas con TIC para el desarrollo del pensamiento computacional en estudiantes de secundaria y primeros ańos de universidad en Iberoamérica (Sarmiento, Gorga, \& Sanz, 2018). Para este artículo, se adopta el termino propuesto por Wings, $(2011$, p.1) el pensamiento computacional es el proceso de pensamiento donde están involucradas la formulación de los problemas y sus soluciones, donde las soluciones están representadas en una forma que se pueden llevar a cabo con eficacia por un agente de procesamiento de información. Con respecto a las características relacionadas con este, los expertos definen diversas características (Figura 1) que identifican al pensamiento computacional (Barr \& Stephenson, 2011; Wing, 2010; Iste \& Csta, 2011; Kemp, 2014; Linn; et al., 2010; Sarmiento, Gorga, \& Sanz, 2016).

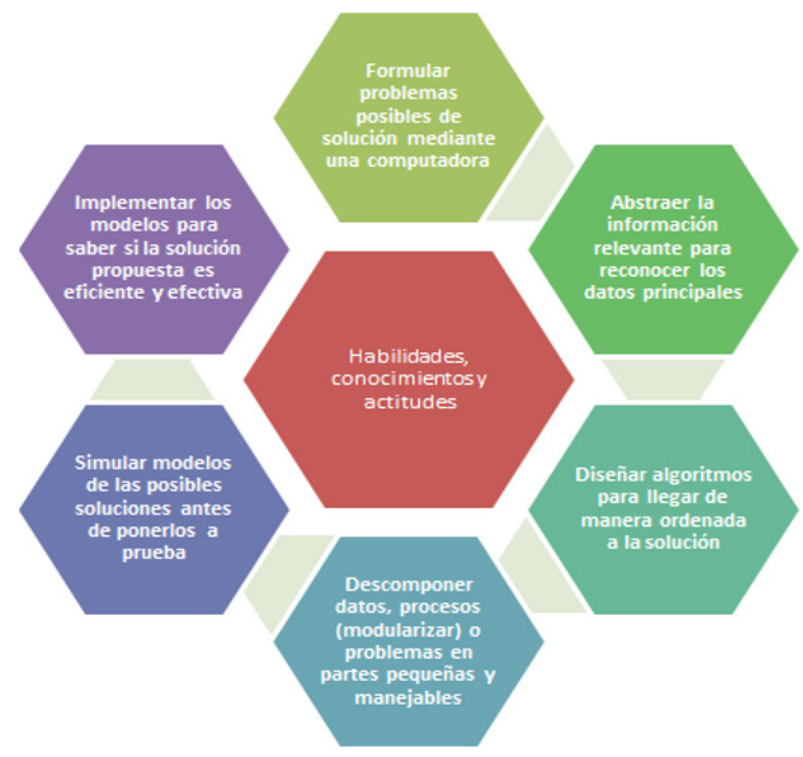

Figura 1. Características del Pensamiento Computacional. Fuente: Elaboración propia

Es necesario señalar que según autoridades en el tema sobre el desarrollo de competencias inherentes al pensamiento computacional (Kafai et al., 2009; Guerrero, Nacional, \& Luis, 2014). Los entornos y lenguajes de programación son herramientas ideales para este tipo de trabajo, es por esto que las experiencias analizadas se centran en la enseńanza de la programación por medio de estas herramientas $u$ otras afines.

\section{Materiales y Métodos}

\section{Participantes}

La muestra seleccionada se realizó considerando que es un estudio bibliográfico y que la información de interés está relacionada con procesos educativos que promueven el desarrollo del pensamiento computacional, siendo los tipos de documentos a indagar actas de congresos, artículos en revistas y portales académicos, capítulos de libros, información directa en los sitios web de las propuestas, capítulos de tesis o reportes de investigación.

\section{Instrumento}

Para la recopilación de información bibliográfica se utilizó un protocolo de búsqueda en el cual se indagó acerca de: conceptos y definiciones sobre pensamiento computacional (PC); habilidades relacionadas con el desarrollo del pensamiento computacional; propuestas educativas que promueven el desarrollo de pensamiento computacional; métodos y herramientas utilizadas para el desarrollo del pensamiento computacional.

Por otro lado, para analizar las experiencias de manera uniforme se contemplaron un conjunto de criterios descriptivos, de los cuales se hace referencia en la Tabla 1.

Tabla 1

Criterios para análisis de experiencias

\section{Criterios descriptivos para el análisis de experiencias}

- Experiencias por país

- Zonas de aplicación: nacional - institucional - áulico

- Ámbitos de aplicación: primario, secundario, universitario, secundario/universitario

- Enfoque para abordar el pensamiento computacional: Solución de problemas académicos o del entorno. Actividades relacionadas con robótica, electrónica, programación o temas a fines

- Herramientas y materiales diseñados

Fuente: Elaboración propia (2019).

Haciendo uso de estos criterios de análisis se reconocen las fortalezas de las propuestas y se logra saber de qué manera abordan el desarrollo de las competencias inherentes al pensamiento computacional. 


\section{Tipo y diseño de estudio}

En esta investigación se realizó un estudio bibliográfico, que utiliza la estrategia de búsqueda sistemática, basada en la etapa de planificación de la revisión propuesta por Barbara Kitchenham (Kitchenham, 2004).

\section{Procedimiento}

El trabajo se realizó en dos momentos, en un primer momento se efectuó la búsqueda de información utilizando la estrategia anteriormente nombrada, para lo cual se siguió un esquema como el que se describe a continuación:

a. Búsqueda de documentos relacionados con el pensamiento computacional por medio de palabras claves, las palabras utilizadas para la búsqueda fueron: pensamiento computacional (PC), conceptos PC, definición PC, desarrollo del PC, experiencias con TIC, integración TIC, enseñanza de programación.

b. Búsqueda de información utilizando fuentes documentales como: librerías digitales, artículos publicados por diversas comunidades científicas, artículos publicados en actas de congresos, repositorios académicos y universitarios, portales académicos, revistas científicas, bases de datos internacionales, uso del buscador Google Academics. Todos los documentos con vigencia de los últimos 10 años de publicación.

c. Aplicación de criterios para considerar la inclusión de documentos: documentos que indagan el concepto de pensamiento computacional, propuestas educativas en las que se fomenten el pensamiento computacional, documentos con propuestas de integración TIC en Iberoamérica, documentos que presentan estándares para la promoción del pensamiento computacional, artículos sobre experiencias de integración en TIC realizadas en escuelas primarias, secundarias y etapas iniciales de universidad, documentos de propuestas sobre enseñanza de la programación.

d. Aplicación de criterios para considerar la exclusión de documentos: se descartaron aquellos artículos con texto incompleto, artículos hallados en fuentes poco confiables, documentos en idiomas que no sean español o inglés, artículos con propuestas de enseñanza de programación que estén fuera del grupo objetivo. $\mathrm{Al}$ realizar la búsqueda respectiva y hacer el proceso preliminar en donde se aplicaron los criterios anteriormente nombrados, se logra un listado de 195 documentos para ser leídos $\mathrm{y}$ analizados. Estos trabajos listados generaron nuevas búsquedas tomando en cuenta las referencias bibliográficas que podían servir para este estudio, de igual manera se indagaron otros escritos de los autores de referencia que tuvieran relación con la temática de interés.

e. Proceso para la selección final del documento: Se aplican los mismos criterios de selección, de allí se obtuvo un resultado de 90 documentos que sirvieron para indagar diversas propuestas utilizadas para el análisis realizado.

f. Herramienta utilizada para la gestión bibliográfica: Mendeley.

En un segundo momento, al terminar la revisión de los 90 documentos se seleccionaron 50 experiencias educativas de las cuales se tomaron 37 para hacer un análisis comparativo, con el objetivo de conocer la manera en que proponían abordar y desarrollar las competencias inherentes al pensamiento computacional, a las 13 restante aunque no se les hizo un análisis a fondo por falta de documentación suficiente para ello, se tuvieron en cuenta porque son antecedentes relacionados al tema de investigación.

\section{Resultados}

A partir de la información recopilada en los documentos encontrados se consideraron 37 propuestas educativas que fueron examinadas y 13 más que son mencionadas en este artículo.

A continuación, se presenta en la Tabla 2 cada una de las propuestas analizadas con su país de origen y la manera en que focalizaron su trabajo, que bien podía ser de manera nacional, institucional o áulico. 
Pensamiento Computacional: Revisión de experiencias y propuestas educativas implementadas en la última década en Iberoamérica

\section{Tabla 2}

Propuestas encontradas y su zona de aplicación

\begin{tabular}{|c|c|c|}
\hline País & Nombre del proyecto & $\begin{array}{l}\text { Zona de } \\
\text { aplicación }\end{array}$ \\
\hline \multirow[t]{13}{*}{ Argentina } & $\begin{array}{l}\text { Astro Código. Un juego serio para } \\
\text { la introducción de jóvenes en los } \\
\text { conceptos básicos de la programación } \\
\text { (La Plata) }\end{array}$ & Institucional \\
\hline & $\begin{array}{l}\text { Construcción de modelos y simulado- } \\
\text { res con Squeak-Etoys como recurso } \\
\text { de aprendizaje en la escuela media } \\
\text { (Rosario) }\end{array}$ & Áulico \\
\hline & $\begin{array}{l}\text { Diseño de un juego basado en Inte- } \\
\text { racción Tangible para la enseñanza de } \\
\text { Programación (La Plata) }\end{array}$ & Institucional \\
\hline & $\begin{array}{l}\text { Desarrollando juegos educativos para } \\
\text { incrementar la participación de los } \\
\text { alumnos en una materia de programa- } \\
\text { ción (La Plata) }\end{array}$ & Institucional \\
\hline & $\begin{array}{l}\text { Enfoque basado en gamificación } \\
\text { para el aprendizaje de un lenguaje de } \\
\text { programación (Santa Rosa) }\end{array}$ & Áulico \\
\hline & $\begin{array}{l}\text { Entrenamiento en la programación de } \\
\text { la computadora a partir de una aplica- } \\
\text { ción para competencias (Neuquén) }\end{array}$ & Institucional \\
\hline & $\begin{array}{l}\text { Herramientas lúdicas como apoyo } \\
\text { a la enseñanza de la programación } \\
\text { (Corrientes) }\end{array}$ & Áulico \\
\hline & $\begin{array}{l}\text { JET: Java en Escuelas Técnicas - } \\
\text { Programando con RITA (La Plata) }\end{array}$ & Institucional \\
\hline & Program.AR & Nacional \\
\hline & Programando con Robots (La Plata) & Institucional \\
\hline & $\begin{array}{l}\text { Programación de videojuegos (Puerto } \\
\text { Madryn) }\end{array}$ & Institucional \\
\hline & Proyecto GEMA (Ituzaingó) & Institucional \\
\hline & Robótica educativa (Tucumán) & Institucional \\
\hline \multirow[t]{2}{*}{ Brasil } & $\begin{array}{l}\text { El uso de Scratch en el desarrollo de } \\
\text { la programación lógica como un aporte } \\
\text { interdisciplinario (Vitoria) }\end{array}$ & Áulico \\
\hline & $\begin{array}{l}\text { Trabajar con la robótica educativa en } \\
\text { la escuela primaria (Amazonas) }\end{array}$ & Institucional \\
\hline \multirow[t]{4}{*}{ Colombia } & CUPI2 (Bogotá) & Institucional \\
\hline & $\begin{array}{l}\text { Robótica e Investigación: Un medio } \\
\text { para la innovación (Medellín) }\end{array}$ & Institucional \\
\hline & $\begin{array}{l}\text { Scratch + ABP, como estrategia para } \\
\text { el desarrollo del pensamiento compu- } \\
\text { tacional(Medellín) }\end{array}$ & Áulico \\
\hline & $\begin{array}{l}\text { Uso de un ambiente virtual competitivo } \\
\text { para el aprendizaje de algoritmos y } \\
\text { programación (Medellín) }\end{array}$ & Institucional \\
\hline $\begin{array}{l}\text { Costa } \\
\text { Rica }\end{array}$ & $\begin{array}{l}\text { PRONIE Programa Nacional de Infor- } \\
\text { mática Educativa }\end{array}$ & Nacional \\
\hline
\end{tabular}

\begin{tabular}{|c|c|c|}
\hline País & Nombre del proyecto & $\begin{array}{l}\text { Zona de } \\
\text { aplicación }\end{array}$ \\
\hline \multirow[t]{6}{*}{ Chile } & $\begin{array}{l}C^{\wedge} 100 \text { Corporación para el Fomento } \\
\text { de la Ciencia de la Computación en } \\
\text { Colegios }\end{array}$ & Nacional \\
\hline & $\begin{array}{l}\text { Desarrollo del Pensamiento computa- } \\
\text { cional con Scratch (Santiago) }\end{array}$ & Institucional \\
\hline & $\begin{array}{l}\text { Experiencias Prácticas con el Uso del } \\
\text { Lenguaje de Programación Scratch } \\
\text { para Desarrollar el Pensamiento } \\
\text { Algorítmico de Estudiantes en Chile } \\
\text { (Viña del Mar) }\end{array}$ & Institucional \\
\hline & $\begin{array}{l}\text { Talleres de robótica para niños y ado- } \\
\text { lescentes, Universidad de Valparaíso } \\
\text { (Valparaíso) }\end{array}$ & Institucional \\
\hline & Taller de Jóvenes Programadores & Nacional \\
\hline & $\begin{array}{l}\text { Un taller de robótica para el apoyo } \\
\text { de la enseñanza de programación de } \\
\text { computadores basado en estilos de } \\
\text { aprendizaje (Valparaíso) }\end{array}$ & Institucional \\
\hline \multirow[t]{7}{*}{ España } & $\begin{array}{l}\text { Uso de Scratch y Lego Mindstorms } \\
\text { como Apoyo a la Docencia en Funda- } \\
\text { mentos de Programación (Valparaíso) }\end{array}$ & Áulico \\
\hline & $\begin{array}{l}\text { Aprendiendo Programación a través } \\
\text { de su enseñanza. Experiencia de } \\
\text { Aprendizaje-Servicio en la universidad } \\
\text { (Madrid) }\end{array}$ & Institucional \\
\hline & Código 21 (Navarra) & Regional \\
\hline & Desafío STEM - Telefónica Fundación & Nacional \\
\hline & mSchools (Cataluña- Barcelona) & Institucional \\
\hline & $\begin{array}{l}\text { Robótica Móvil y Programación en } \\
\text { Educación Secundaria, Robocampeo- } \\
\text { nes (Fuentelabrada) }\end{array}$ & Institucional \\
\hline & Xnergic (Barcelona) & Institucional \\
\hline \multirow[t]{2}{*}{ Ecuador } & Scientific Kids University (Quito) & Institucional \\
\hline & Robótica educativa (Soyapango) & Áulico \\
\hline $\begin{array}{l}\text { El Salva- } \\
\text { dor }\end{array}$ & $\begin{array}{l}\text { Los Mundos Virtuales: una plataforma } \\
\text { para el desarrollo de habilidades de } \\
\text { programación y de interacción social } \\
\text { (Lima) }\end{array}$ & Institucional \\
\hline Perú & $\begin{array}{l}\text { Butiá: Plataforma robótica genérica } \\
\text { para la enseñanza de la informática } \\
\text { (Uruguay) }\end{array}$ & Nacional \\
\hline
\end{tabular}

Fuente: Contenidos resumidos de Artola et al.,(2014); Astudillo, Bast \& Willging,(2016); Capot \& Espinoza, (2015); Damian et al., (2015); Damian, Defosse \& Salvatierra, (2013); Feijoo et al., (2010); Harari \& Tzancoff, (2014); López, Muñoz \& Barría, (2012); Moreno \& Montoya, (2013); Muñoz et al., (2015); Queiruga et al., (2016); Restrepo, (2013); Rosales et al., (2015); Tejera, Andrade, 
\& Gindel,(2011); Tzancoff, \& Queiruga, (2014); Vidal et al., (2015).

Según el análisis realizado a esta información, se encontró que (Tabla 3): los países con mayor cantidad de experiencias halladas son Argentina (35\%) y Chile (19\%), seguidas de España (16\%) y Colombia (11\%). Las propuestas que se tuvieron en cuenta para este análisis como se dijo anteriormente no son las únicas, pero son aquellas que presentan información suficiente en cuanto a la descripción del trabajo realizado, objetivos, estrategias, métodos, implementación, resultados, para poder realizar el análisis respectivo.

Tabla 3

Experiencias por países

\begin{tabular}{lc}
\hline \multicolumn{1}{c}{ País } & Propuestas \% \\
\hline Argentina & 35 \\
\hline Chile & 19 \\
\hline España & 16 \\
\hline Colombia & 11 \\
\hline Brasil & 5 \\
\hline Costa Rica & 2 \\
\hline El Salvador & 3 \\
\hline Ecuador & 3 \\
\hline Perú & 3 \\
\hline Uruguay & 3 \\
\hline
\end{tabular}

Fuente: Elaboración propia (2019).

Así mismo se encuentra que porcentualmente las zonas en las cuales se aplican los proyectos están distribuidas de la siguiente manera: institucionalmente en un $62 \%$, un $19 \%$ difundió las propuestas a nivel nacional o regional y otro 19\% trabajó proyectos áulicos (Tabla 4).

\section{Tabla 4}

Zonas de aplicación

\begin{tabular}{lc}
\hline Zona de aplicación & Propuestas \% \\
\hline Institucional & 62 \\
\hline Nacional - regional & 19 \\
\hline Áulico & 19 \\
\hline
\end{tabular}

Fuente: Elaboración propia (2019).

Continuando con el análisis realizado, se encontró que algunas de las propuestas, además de desarrollar el pensamiento computacional, promueven estrategias para solucionar problemas académicos como la deserción escolar, la dificultad en el aprendizaje o la falta de material educativo, de igual manera buscan satisfacer necesidades de su entorno como brindar ayuda a personas con discapacidad, disminuir la brecha educativa o incentivar vocaciones tecnológicas. Por otro lado se observa que las propuestas se implementan en ámbitos de primaria, secundaria, a nivel universitario y universitario - secundario integrado, centrándose en los proyectos de secundaria y universidad debido a que las facultades se interesan en generar propuestas de articulación entre las instituciones, con el fin de facilitar la transición desde los establecimientos educativos de secundaria hacia las universidades y de este modo favorecer la calidad de los egresados de la escuela media (Queiruga \& Fava, 2014).

En la Tabla 5, se muestra el enfoque desde el cual cada propuesta pretende desarrollar el pensamiento computacional, junto con el ámbito de implementación.

Tabla 5

Enfoque de la propuesta y ámbitos de aplicación

\begin{tabular}{lll}
\hline \multicolumn{1}{c}{ Propuestas y Experiencias } & $\begin{array}{c}\text { Tipo de estra- } \\
\text { tegia }\end{array}$ & $\begin{array}{l}\text { Nivel de apli- } \\
\text { cación }\end{array}$ \\
\hline Robótica educativa (Argentina) & & Secundario \\
\hline $\begin{array}{l}\text { Robótica educativa (El } \\
\text { Salvador) }\end{array}$ & Secundario \\
\hline Robótica e Investigación: Un & & Secundario \\
medio para la innovación & & \\
\hline $\begin{array}{l}\text { Astro Código. Un juego serio } \\
\text { para la introducción de jóvenes }\end{array}$ & $\begin{array}{l}\text { Desarrollo del } \\
\text { pensamiento }\end{array}$ & Secundario \\
en los conceptos básicos de la & computacio- \\
programación & nal desde & \\
& $\begin{array}{l}\text { la robótica, } \\
\text { electrónica, } \\
\text { programación }\end{array}$ \\
& o temas a & \\
& fines & \\
\hline $\begin{array}{l}\text { Aprendiendo Programación a } \\
\text { través de su enseñanza. Expe- } \\
\text { riencia de Aprendizaje-Servicio } \\
\text { en la universidad }\end{array}$ & & Secundario \\
\hline $\begin{array}{l}\text { Butiá: Plataforma robótica } \\
\text { genérica para la enseñanza de } \\
\text { la informática }\end{array}$ & & Secundario \\
\hline en la escuela medigo 21 & & Secundario \\
\hline Construcción de modelos y & & \\
\hline
\end{tabular}


Pensamiento Computacional: Revisión de experiencias y propuestas educativas implementadas en la última década en Iberoamérica

\begin{tabular}{|c|c|c|}
\hline Propuestas y Experiencias & $\begin{array}{l}\text { Tipo de estra- } \\
\text { tegia }\end{array}$ & $\begin{array}{l}\text { Nivel de apli- } \\
\text { cación }\end{array}$ \\
\hline Desafío STEM & & Secundario \\
\hline $\begin{array}{l}\text { Desarrollo del Pensamiento } \\
\text { computacional con Scratch }\end{array}$ & & Sec//Un \\
\hline $\begin{array}{l}\text { Entrenamiento en la progra- } \\
\text { mación de la computadora a } \\
\text { partir de una aplicación para } \\
\text { competencias }\end{array}$ & & $\begin{array}{l}\text { Secundario/ } \\
\text { Universitario }\end{array}$ \\
\hline $\begin{array}{l}\text { Experiencias Prácticas con } \\
\text { el Uso del Lenguaje de Progra- } \\
\text { mación Scratch para Desarro- } \\
\text { llar el Pensamiento Algorítmico } \\
\text { de Estudiantes en Chile }\end{array}$ & & Secundario \\
\hline $\begin{array}{l}\text { Los Mundos Virtuales: una pla- } \\
\text { taforma para el desarrollo de } \\
\text { habilidades de programación y } \\
\text { de interacción social }\end{array}$ & & Universitario \\
\hline mSchools & & Secundario \\
\hline Programando con Robots & & Sec/Un \\
\hline Programación de videojuegos & & Sec/UN \\
\hline $\begin{array}{l}\text { Programa Nacional de Infor- } \\
\text { mática Educativa PRONIE }\end{array}$ & & Secundario \\
\hline $\begin{array}{l}\text { Robótica Móvil y Programa- } \\
\text { ción en Educación Secundaria, } \\
\text { Robocampeones }\end{array}$ & & Secundario \\
\hline $\begin{array}{l}\text { Scratch + ABP, como estra- } \\
\text { tegia para el desarrollo del } \\
\text { pensamiento computacional }\end{array}$ & & Secundario \\
\hline Scientific Kids University & & Sec/UN \\
\hline $\begin{array}{l}\text { Taller de Jóvenes Programa- } \\
\text { dores }\end{array}$ & & Secundario \\
\hline $\begin{array}{l}\text { Talleres de robótica para niños } \\
\text { y adolescentes, Universidad } \\
\text { de Valparaíso }\end{array}$ & & Sec//Un \\
\hline $\begin{array}{l}\text { Trabajar con la robótica educa- } \\
\text { tiva en la escuela primaria }\end{array}$ & & Primario \\
\hline $\begin{array}{l}\text { Uso de Scratch y Lego } \\
\text { Mindstorms como Apoyo a la } \\
\text { Docencia en Fundamentos de } \\
\text { Programación }\end{array}$ & & Universitario \\
\hline $\begin{array}{l}\text { Uso de un ambiente virtual } \\
\text { competitivo para el aprendizaje } \\
\text { de algoritmos y programación }\end{array}$ & & Universitario \\
\hline $\begin{array}{l}\text { Un taller de robótica para el } \\
\text { apoyo de la enseñanza de } \\
\text { programación de computa- } \\
\text { dores basado en estilos de } \\
\text { aprendizaje }\end{array}$ & & Universitario \\
\hline Xnergic & & Secundario \\
\hline
\end{tabular}

Montoya, (2013); Muñoz et al., (2015); Queiruga et al., (2016); Restrepo, (2013); Rosales et al., (2015); Tejera, Andrade, \& Gindel,(2011); Tzancoff, \& Queiruga, (2014); Vidal et al., (2015).

Teniendo en cuenta la información revisada, se encuentra que el $70 \%$ de las propuestas utilizan la robótica, electrónica, programación o temas relacionados para fomentar el pensamiento computacional y aquellas que lo abordan desde la solución de problemas diversos son el $30 \%$ como se presenta en la Tabla 6.

Tabla 6

Desarrollo del pensamiento computacional desde distintos enfoques

\begin{tabular}{lc}
\hline $\begin{array}{l}\text { Enfoque para fomentar el pensamiento } \\
\text { computacional }\end{array}$ & Propuestas \% \\
\hline $\begin{array}{l}\text { Desde la electrónica, robótica, } \\
\text { programación y temas a fines }\end{array}$ & 70 \\
\hline $\begin{array}{l}\text { Resolución de problemas académicos o } \\
\text { del entorno }\end{array}$ & 30 \\
\hline
\end{tabular}

Fuente: Elaboración propia (2019).

Por otra parte, se encuentra que las propuestas implementadas en el ámbito primario son del $3 \%$, en el ámbito universitario del $22 \%$ y las implementadas en un ámbito universitario - secundario son de $24 \%$, el ámbito con mayor porcentaje es el secundario con un 51\% (Tabla 7). Como se mencionó anteriormente esto se debe al interés de las instituciones por lograr la calidad de los egresados y favorecer la continuidad de los estudiantes de secundaria en carreras universitarias relacionadas con esta área del conocimiento.

Tabla 7

Ámbitos de aplicación

\begin{tabular}{lc}
\hline \multicolumn{1}{c}{ Ámbito } & Propuestas \% \\
\hline Secundario & 51 \\
\hline Universitario y secundario & 24 \\
\hline Universitario & 22 \\
\hline Primario & 3 \\
\hline
\end{tabular}

Fuente: Elaboración propia (2019).

Con respecto a las herramientas y materiales utilizados se encontró que la mayoría de propuestas desarrollaron hardware, software o entornos, junto con los materiales necesarios, guías, manuales, tutoriales, sitios web, para la aplicación de sus es-

Fuente: Los contenidos fueron resumidos de Artola et al., (2014); Astudillo, Bast \& Willging,(2016); Capot \& Espinoza, (2015); Damian et al., (2015); Damian, Defosse \& Salvatierra, (2013); Feijoo et al., (2010); Harari \& Tzancoff, (2014); López, Muñoz \& Barría, (2012); Moreno \& 
trategias educativas. En la Figura 2 se nombran las propuestas que desarrollaron plataforma y material documental, junto con las que diseñaron el software y hardware utilizado.

\section{Plataforma virtual y material documental}

- C^100 Corporación para el Fomento de la Ciencia de la Computación en Colegios

- Código21 CUPI2

- Desafío STEM

- mSchools

- Program.AR

- Programa Nacional de Informática Educativa PRONIE

- Uso de un ambiente virtual competitivo para el aprendizaje de algoritmos y programación

- Robótica Móvil y Programación en Educación Secundaria Robocampeones

- Un taller de robótica para el apoyo de la enseñanza de programación de computadores basado en estilos de aprendizaje

\section{Hardware, software y materiales}

- AstroCódigo. Un juego serio para la introducción de jóvenes en los conceptos básicos de la programación.

- Butiá: Plataforma robótica genérica para la enseñanza de la informática

- Diseño de un juego basado en Interacción Tangible para la enseñanza de Programación

- Entrenamiento en la programación de la computadora a partir de una aplicación para competencias

- J ET: Java en Escuelas Técnicas, Programando con RITA

- Programando con Robots

- Robótica educativa

Figura 2. Herramientas y materiales propios diseñados para las propuestas educativas

Fuente: elaboración propia (2019).

Teniendo en cuenta la información analizada se encontró que 17 de las propuestas hacen uso de materiales y herramientas con diseño propios, junto con software libre y gratuito, las demás experiencias hacen uso de software y hardware de autor, combinado con software libre y gratuito.

El aporte que se encontró según la revisión realizada, está relacionado con la manera en que estas experiencias integran las TIC de forma eficaz en los procesos educativos, con el fin de fomentar habilidades relacionadas con el pensamiento computacional, las propuestas analizadas adaptan las actividades y sus contenidos teniendo en cuenta el contexto donde se desarrollan, para hacer uso de herramientas de software y hardware adecuadas al medio y a las necesidades. Por otro lado, se observa que cada una de ellas pretenden abordar el desarrollo de las habilidades, conocimientos y actitudes inherentes al pensamiento computacional mencionadas al inicio de este artículo.
A continuación, se presentan las propuestas consideradas, pero que no fueron incluidas en el análisis que se muestra en este artículo por la poca información que se encontró referente a ellas, cabe añadir que aunque no se incluyen en las anteriores tablas, son importantes en el momento de reconocer que algunas de ellas son pioneras en la integración de las TIC en Iberoamérica y se debe continuar con la búsqueda de información publicada recientemente donde se haga referencia a su implementación y a sus resultados, de modo que contribuya a complementar el estudio de esta temática.

\section{Proyectos en los que no se conocen características}

de la implementación y resultados

- Roboeduca, red de robótica educativa (Argentina)

- Daleacepta (Argentina)

- Desarrollo de un lenguaje de programación y entorno de desarrollo que facilite la programación de robots LEGO mindstorms (Chile)

- Concurso Latinoamericano de Proyectos Estudiantiles de Ciencia y Tecnología

- Pensamiento Computacional a través de la Programación: Paradigma de Aprendizaje (España)

- ProBot: Juego para el aprendizaje de lógica de Programación (Colombia)

- Proyecto Robótica Montevideo Oeste Incorporación de la Robótica en

Escuelas de TC (URUGUAY)

- Proyecto Descubre (España)

Proyectos nacionales en donde no se especifica las

estrategias y métodos utilizados en la implementación

- Plan Nacional Integral de Educación Digital - PLANIED Argentina)

- Computadores Para Educar-CPE (Colomba)

- Programa "Cerrando la brecha del conocimiento" (El Salvador)

- Plan Ceibal (Uruguay)

- Proyecto Enlaces(Chiie)

Figura 3. Propuestas no incluidas en las tablas de análisis anteriores

Fuente: elaboración propia (2019).

\section{Discusión y Conclusiones}

La recopilación de información realizada permitió indagar, revisar y describir aspectos referentes a una serie de propuestas implementadas alrededor de Iberoamérica con el fin de desarrollar las competencias inherentes al pensamiento computacional.

A partir del estudio elaborado se reconocen características específicas de cada una de las experiencias, de este modo se brinda información relacionada con las estrategias utilizadas en su implementación, aspectos que pueden orientar a instituciones o docentes para la generación y dise- 
Pensamiento Computacional: Revisión de experiencias y propuestas educativas implementadas en la última década en Iberoamérica

ño de nuevas propuestas que vinculen este tipo de temática. Se puede observar que el país que brinda mayor información acerca de la puesta en marcha y la estrategia utilizada en las experiencias es Argentina, seguida de Chile y España. Además, se encontró que las propuestas son implementadas de manera nacional o regional, también en aula, pero sobre todo de manera institucional y se desarrollan en niveles de secundaria con el objetivo de fortalecer el pensamiento computacional para así lograr una adecuada transición de los estudiantes desde la escuela a la universidad. Por otro lado, se halló que las estrategias utilizadas para abordar las competencias inherentes al pensamiento computacional son diversas y entre ellas están la solución de problemas o satisfacción de necesidades académicas y del entorno, junto con el manejo de herramientas robóticas, electrónicas y de programación. De igual manera se halló que la mitad de las propuestas diseñan y desarrollan sus propios materiales y herramientas tanto hardware como software, complementándolas para algunas actividades con herramientas de autor.

Este artículo permite conocer ciertas características de una serie de propuestas y experiencias, cabe resaltar que no son las únicas y es importante continuar indagando acerca de ellas, puesto que no se debe olvidar la constante evolución y el interés que despierta esta temática en el entorno educativo, motivo por el cual es importante continuar trabajando esta línea de investigación hasta precisar cuáles son las estrategias que ofrecen mejores resultados y la manera adecuada de abordar el desarrollo del pensamiento computacional.

Aunque en el artículo se nombran programas nacionales para la integración de tecnologías que tienen más de 10 años de implementación, es importante tenerlos en cuenta porque han sido pioneros en el diseño de estrategias para incorporar las TIC en el aula y lograr el desarrollo de habilidades en el uso de dispositivos tecnológicos tanto de estudiantes como docentes de las instituciones públicas en cada uno de los países.

La revisión que se hace en este artículo ofrece la oportunidad de reconocer algunas características de las estrategias y métodos utilizados en las propuestas estudiadas, que pueden ser adaptadas al entorno educativo en el que se desea implementar y de esta manera generar nuevas experiencias en pro del desarrollo del pensamiento computacional.

\section{REFERENCIAS BIBLIOGRÁFICAS}

Artola, V., Sanz, C., Gorga, G. \& Pesado, P. (2014). Diseño de un juego basado en Interacción Tangible para la enseñanza de Programación (Tesis de pregrado). Universidad Nacional de la Plata, Argentina. Recuperado de http://sedici.unlp. edu.ar/handle/10915/42131

Astudillo, G. J., Bast, S. G. \& Willging, P. A. (2016). Enfoque basado en gamificación para el aprendizaje de un lenguaje de programación. Virtualidad, Educación y Ciencia, (12), 125-142. Recuperado de https://revistas.unc.edu.ar/ index.php/vesc/article/view/14739

Barr, V. \& Stephenson, C. (2011). Bringing Computational Thinking to K-12: What is Involved and What is the Role of the Computer Science Education Community. ACM Inroads, 2(1), 48-54. https://doi. org/10.1145/1929887.1929905

Capot, R. B. \& Espinoza, R. M. (2015). Desarrollo del Pensamiento Computacional con Scratch. En J. Sánchez (Presidente), Nuevas ideas en informática educativa. Simposio llevado a cabo en el XX Congreso internacional de Informática Educativa, Santiago, Chile.

Da Silva, A., Da Silva Martins, L. A., Ramos Nascimento, G. \& Do Santos Marints, thais. (2016). Trabalhando Com Robótica Educacional No Ensino Fundamental. En J. Sánchez (Presidente), TISE 2016. Simposio llevado a cabo en el XXI Congreso Internacional de Informática Educativa, Santiago, Chile.

Damian, B., Stickar, R., Defosse, N. \& Cura, R. (2015). Programación de videojuegos en el Nivel Medio. En G. Dapozo (Presidente), Simposio llevado a cabo en el X Congreso de Tecnología en Educación \& Educación en Tecnología, Corrientes, Argentina.

Damian, B., Stickar, R., Defosse, N. \& Salvatierra, S. (2013). Programación de videojuegos en el nivel medio. Simposio llevado a cabo en el Congreso Iberoamericano de Ciencia y Tecnología, Buenos Aires, Argentina.

Dapozo, G., Greiner, C. \& Petris, R. (2016). Herramientas lúdicas como apoyo a la enseńanza de la programación. En G. Feierherd (Coordinador), CACIC2016. Simposio llevado a cabo en el XXII Congreso Argentino de Ciencias de la Computación, San Luis, Argentina.

Feijoo, A., Baldeón, J., Nakano T., \& Molla I. (2010). Los Mundos Virtuales: una plataforma para el desarrollo de habilidades de programación y de interacción social. Recuperado de http://www.virtualeduca.info/fveduca/es/tematica/42-la-universidad-en-lasociedad-del-conocimiento-/146-los-mundos-virtuales-una-plataformapara-el-desarrollo-de-habilidades-de-programacion-y-de-interaccionsocial 
Guerrero, R. A., Nacional, L.U. \& Luis, D. S. (2014). El Desarrollo del Pensamiento Computacional para la Resolución de Problemas en la Enseńanza Inicial de la Programación. WICC 2014. Simposio llevado a cabo en el XVI Workshop de Investigadores en Ciencias de la Computación, Ushuaia, Argentina.

Harari, V. \& Tzancoff, C. (2014). Desarrollando juegos educativos para incrementar la participación de los alumnos en una materia de programación. En D. Pulfer (Presidencia) , Simposio llevado a cabo en el Congreso Iberoamericano de Ciencia, Tecnología, Innovación y Educación, Buenos Aires, Argentina.

Iste. Csta. (2011). Computational Thinking in K-12 Education leadership toolkit. 43. Recuperado de http://www. iste.org/learn/computational-thinking

Kafai, Y., Resnick, M., Maloney, J., Monroy-Hernández, A., Rusk, N., Eastmond, E., \& Silverman, B. (2009). Scratch: Programming for All. Communications of the ACM, 52, 60-67. https://doi.org/10.1145/1592761.1592779

Kemp, P. (2014). Computing in the national curriculum A guide for secondary teachers Computing in the. Computing at School. Ed. Recuperado de http://www.computingatschool.org.uk/data/uploads/cas_secondary.pdf

Kitchenham, B. (2004). Procedures for performing systematic reviews. Keele University, 33(TR/SE-0401), 28. Recuperado de http://www.inf.ufsc.br/ aldo.vw/kitchenham.pdf

Linn;, M. C., Aho;, A. V, Blake;, M. B., Constable;, R., Kafai;, Y. B., Kolodner;, \& J. L., Bradley, S. (2010). Report of a Workshop on The Scope and Nature of Computational Thinking. https://doi.org/10.17226/12840

López, R. N., Muñoz, R. \& Barría, M. (2012). Un taller de robótica para el apoyo de la enseńanza de programación de computadores basado en estilos de aprendizaje. En J. Sanchez (Presidencia), Nuevas ideas en informática educativa. Simposio llevado a cabo en el XVII Congreso Internacional de Informática Educativa, Santiago, Chile.

Moreno, J., \& Montoya, L. F. (2013). Uso de un ambiente virtual competitivo para el aprendizaje de algoritmos y programación. En M. De Borba (Coordinadora), TISE 2013. Simposio llevado a cabo en el XVIII Congreso Internacional de Informática Educativa, Porto Alegre, Brasil.

Muñoz, R., Barcelos, T. S., Villarroel, R., Barría, M., Becerra, C., Noel, R. \& Silveira, I. F. (2015). Uso de Scratch y Lego Mindstorms como Apoyo a la Docencia en Fundamentos de Programación. En M. Castaño (presidencia), JENUI 2015.Simposio llevado a cabo en el XXI Jornadas de La Enseñanza Universitaria de Informática, Andorra La vella, Principado de Andorra.

Queiruga, C., Fava, L., Banchoff, C., Aybar, V. del C., Kimura, I. M. \& Brown, M. (2016). RITA: una herramienta didáctica-pedagógica innovadora en la escuela secundaria. Recuperado de https://jets.linti.unlp.edu.ar/uploads/docs/ rita_una_herramienta_didactica_pedagogica_innovadora_en_la_escuela_secundaria.pdf

Queiruga, C., Fava, L., Gómez, S., Kimura, I. M., \& Bart- neche, B. (2014). El juego como estrategia didáctica para acercar la programación a la escuela secundaria JET: Java en Escuelas Técnicas secundaria, En Red UNCI (Coordinador), WICC 2014. Simposio llevado a cabo en el XVI Workshop de Investigadores en Ciencias de la Computación, Ushuaia, Argentina.

Restrepo, E. (2013). Robótica e Investigación: Un medio para la innovación Experiencia de robótica educativa e Investigación en el Colegio Montessori- Medellín, 1-15. Recuperado de http://reposital.cuaed.unam.mx:8080/jspui/ bitstream/123456789/3684/1/VE13.227.pdf

Rios, G. C. (2015). Scratch + ABP, Como estrategia para el desarrollo del Pensamiento Computacional (Tesis de maestría), Universidad EAFIT. Recuperado de

https://repository.eafit.edu.co/bitstream/handle/10784/7849/GloriaCecilia_RiosMu\%c3\%b1oz_2015. pdf? sequence $=2 \&$ isAllowed $=y$

Rosales, V. A., Queiruga, C., Kimura, I. M., Barnetche, M. B. \& Goméz, S. (2015). Enseñando a programar con RITA en escuelas secundarias. Recuperado de http://sedici.unlp. edu.ar/bitstream/handle/10915/50644/Documento_completo.pdf-PDFA.pdf?sequence=

Sarmiento, M. S., Sanz, C. \& Gorga, G. (2018). Diseño de una propuesta metodológica para el desarrollo de competencias relacionadas con el pensamiento computacional (tesis de maestría). Universidad Nacional de La Plata UNLP. La Plata, Argentina.

Sarmiento, M., Gorga, G. \& Sanz, C. (2016). Análisis de experiencias y estrategias educativas con TIC para el desarrollo del pensamiento computacional en estudiantes de secundaria y primeros años de universidad en Iberoamérica (tesis de especialización). Universidad Nacional de La Plata UNLP, La Plata, Argentina.

SITEAL. (2014). Informe de tendencias sociales y educativas en América Latina 2014. Siteal, 1-262. Recuperado de http://www.siteal.iipe.unesco.org/sites/default/files/siteal_ informe_2014_politicas_tic.pdf

Tejera, G., Andrade, F., \& Gindel, P. (2011). Butiá: Plataforma robótica genérica para la enseñanza de la informática. En A. Lutemberg (Presidente), CASE 2011. Simposio llevado a cabo en el Congreso Argentino de Sistemas Embebidos, Buenos Aires, Argentina.

Tzancoff, B., \& Queiruga, C. (2014). Experiencias de la Facultad de Informática en la Enseñanza de Programación en Escuelas con Software Libre. En D. Pulfer (Presidencia), Simposio llevado a cabo en el Congreso Iberoamericano de Ciencia, Tecnología, Innovación y Educación, Buenos Aires, Argentina.

Vidal, C. L., Cabezas, C., Parra, J. H., \& López, L. P. (2015). Experiencias Prácticas con el Uso del Lenguaje de Programación Scratch para Desarrollar el Pensamiento Algorítmico de Estudiantes en Chile. Formación Universitaria, 8(4), 23-32. https://doi.org/10.4067/S0718-50062015000400004

Wing, J. M. (2010). Computational Thinking: What and 
Pensamiento Computacional: Revisión de experiencias y propuestas educativas implementadas en la última década en Iberoamérica

Why?. Thelink - The Magaizne of the Carnegie Mellon University School of Computer Science. Recuperado de http://www.cs.cmu.edu/link/research-notebook-computational-thinking-what-and-why 